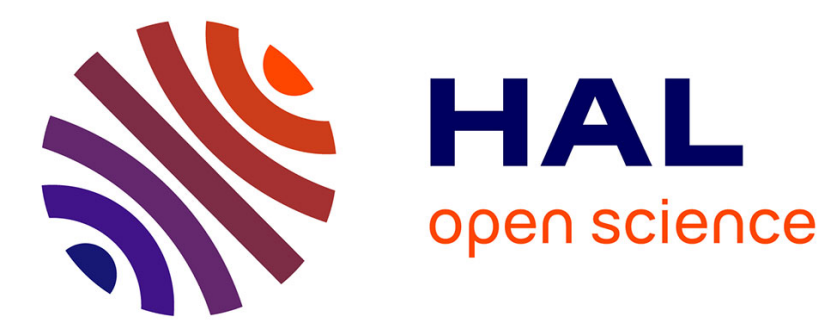

\title{
Classical and ab Initio Plasmonics Meet at Sub-nanometric Noble Metal Rods
}

Rajarshi Sinha-Roy, Pablo García-González, Hans-Christian Weissker, Franck Rabilloud, Antonio Fernández-Domínguez

\section{- To cite this version:}

Rajarshi Sinha-Roy, Pablo García-González, Hans-Christian Weissker, Franck Rabilloud, Antonio Fernández-Domínguez. Classical and ab Initio Plasmonics Meet at Sub-nanometric Noble Metal Rods. ACS photonics, 2017, 4 (6), pp.1484 - 1493. 10.1021/acsphotonics.7b00254 . hal-01784859

\section{HAL Id: hal-01784859 \\ https://hal-amu.archives-ouvertes.fr/hal-01784859}

Submitted on 4 May 2018

HAL is a multi-disciplinary open access archive for the deposit and dissemination of scientific research documents, whether they are published or not. The documents may come from teaching and research institutions in France or abroad, or from public or private research centers.
L'archive ouverte pluridisciplinaire HAL, est destinée au dépôt et à la diffusion de documents scientifiques de niveau recherche, publiés ou non, émanant des établissements d'enseignement et de recherche français ou étrangers, des laboratoires publics ou privés. 


\title{
Classical and Ab Initio Plasmonics Meet at Sub-nanometric Noble Metal Rods
}

\author{
Rajarshi Sinha-Roy, ${ }^{*, \dagger},+\S$ Pablo García-González, ${ }^{\dagger, \S}$ Hans-Christian Weissker, ${ }^{\dagger, \S}$ \\ Frank Rabilloud, `and Antonio I. Fernández-Domínguez*,‡ \\ $\dagger$ Centre Interdisciplinaire de Nanoscience de Marseille (CINaM), Aix-Marseille University, \\ 13288 Marseille, France \\ $\ddagger$ Departamento de Física Teórica de la Materia Condensada and Condensed Matter \\ Physics Center (IFIMAC), Universidad Autónoma de Madrid, E-28049 Madrid, Spain \\ ๆ Univ Lyon, Université Claude Bernard Lyon 1, CNRS, Institut Lumière Matière, \\ F-69622, VILLEURBANNE, France \\ $\S$ European Theoretical Spectroscopy Facility (ETSF) \\ E-mail: sinharoy@cinam.univ-mrs.fr; a.fernandez-dominguez@uam.es
}

\begin{abstract}
Applications of noble metal clusters and nanoparticles in different size ranges abound — from a couple of atoms through mesoscopic sizes. Classical electromagnetics calculations are now employed on smaller and smaller sizes, creating a effervescent dynamic in fields like plasmonics and approaching the tiny sizes where quantum effects and the atomistic structure of matter play predominant roles. Nonetheless, explicit demonstrations of their merits and limitations are rare. Here we study the optical absorption of sub-nanometric elongated coinage-metal particles using ab initio and classical electromagnetics methods. The comparison between both approaches reveals that the classical plasmonic frequencies are in astonishing agreement with those predicted by
\end{abstract}


ab initio theory for atomistic three-dimensional rods and quasi one-dimensional chains, as long as collective surface-plasmon resonances lie far below the onset of $d$-electron excitations. The physical origin of this striking agreement is clarified through the analysis of the resonant induced electron densities and with the aid of model calculations for a purely one-dimensional system of electrons. Furthermore, we show that even when plasmonic/collective and electron-hole excitations are strongly coupled, the classical description accounts rather well for the spectral average of the corresponding quantum hybrid excitations. Our theoretical findings demonstrate that classical optics is surprisingly accurate in describing localized surface plasmon resonances even for angstrom-sized systems, provided the geometrical modelling of the atomistic structures is properly defined.

\section{Keywords}

Localized Plasmon Resonance, d-electron Excitations, Time-Dependent Density Functional Theory, Ab Initio Methods, Classical Optics, Nanorod, Atomic Chain

Localized Surface-Plasmon Resonances (LSPRs) in noble metal nanoparticles are receiving enormous research attention due to their numerous applications in biological sensing, ${ }^{1}$ surface-enhanced Raman spectroscopy (SERS), ${ }^{2}$ cancer therapy, ${ }^{3}$ detection technologies ${ }^{4}$ and also as efficient light-harvesting agents. ${ }^{5}$ The main properties that make LSPRs essential for these applications are their ability to confine and enhance light down to the nano-scale, their design flexibility through size and geometry, and their highly sensitive dependence on the environment. 6,7

Over the years, classical optics has been customarily used to investigate LSPRs in large (sizes greater than $10 \mathrm{~nm}$ ) noble-metal nanoparticles. Among the broad range of struc-

tures explored, special attention has been focused on elongated particles, or nanorods, ${ }^{8-10}$ in which LSPR frequencies can be tuned through the geometrical aspect ratio (AR, defined 
as the ratio between the longitudinal and lateral dimensions of the system). However, small clusters of noble metals ${ }^{11-13}$ and ultrathin rods with diameters smaller than $2 \mathrm{~nm}^{14}$ can be synthesized as well. Also, a number of stable thiolate protected Au clusters ${ }^{15-17}$ can be formed, among which $\mathrm{Au}_{144}(\mathrm{SR})_{60}$ has appeared to be of utmost interest due to its size $(\simeq 1.8 \mathrm{~nm})$, stability, and coherent reproducibility of optical spectra. ${ }^{18,19}$ These advances open up a different length scale for plasmonics, where genuine quantum effects like quantization of electron energy levels, electron-density spill-out, and reduced surface $d$-electron screening become important. An example of the peculiar LSPR phenomenology emerging in this regime is the suppression of transverse plasmonic modes due to the quantization of electron energy levels in ultrathin Au rods. ${ }^{14}$ As anticipated above, apart from their robustness against purely quantum physical effects, LSPRs in nanostructures present another very interesting optical characteristic: their spectral tunability through the nanostructure AR. ${ }^{8,20}$ In particular, as recently reported for Au rods, ${ }^{21,22}$ this paves the way to controlling the coupling between LSPRs and $d$-electron excitations through geometry. Moreover, when the width of the elongated nanostructure reaches a critical value, the electron motion is practically confined along the rod axis. In this regime, the system cannot be considered as a three-dimensional one and, as a consequence, collective electron excitations can no longer be considered as surface modes. ${ }^{23}$

Lately, there has been an increasing interest in the modelling of the LSPR response of tiny noble metal structures by electromagnetics tools. Thus, it has been recently shown that sub-nanometric inhomogeneities in field-enhancement patterns in plasmonic gaps ${ }^{24-26}$ can be described, at least qualitatively, through classical optics calculations. On the other hand, the physical mechanisms behind the failure of classical predictions for the LSPR frequencies in compact nanoparticles (with sizes in the $\sim 1-3 \mathrm{~nm}$ range) are well understood. ${ }^{11,12,27-29}$ Nonetheless, a careful exploration of the actual capabilities and limitations of electromagnetics methods when applied to elongated noble-metal subnanometric structures is, to the best of our knowledge, still lacking. In order to fill this gap, we present a comparative study 
of $a b$ initio and classical optics (local and nonlocal) descriptions of the optical properties of noble-metal (Ag and $\mathrm{Au}$ ) rods and chains of different sizes and ARs.

We develop a simple and intuitive link between quantum and classical descriptions of LSPRs, which allows us to define geometries (both shapes and dimensions) for the electromagnetics calculations in correspondence with atomistic structures having sub-nanometric lateral dimensions. Subsequently, we demonstrate the astonishing quality of the AR dependence of LSPRs as calculated by classical means in these quantum-sized systems, even in the quasi-one-dimensional limit. A direct comparison of LSPR frequencies and spectral strengths by both classical and $a b$ initio descriptions reveals the influence of $d$-electron transitions in the plasmonic response of low-AR (quasi-spherical) structures. Finally, we investigate induced LSPR charge densities from classical and TDDFT calculations. This allows us to extend our comparative study beyond far-field characteristics and verify the validity of our findings also in the near-field regime. Apart from its evident methodological implications, our theoretical findings shed light on the interplay between collective and single-electron excitations in metallic nanostructures, a topic which is attracting much attention currently in various fields, including plasmonic-enhanced hot electron generation, ${ }^{30}$ photocatalysis ${ }^{31}$ and nano-scale heat sources. ${ }^{32}$

Theoretical background. There is a wide range of tools for the theoretical description of LSPRs. The most suitable approach varies from case to case, depending on the geometry and size of the metallic structure and its environment. From an ab initio perspective, timedependent density-functional theory (TDDFT) ${ }^{33}$ is the method of choice to study collective excitations in metallic nanostructures. ${ }^{21,29,34-38}$ In TDDFT, the time evolution of the density of the many-electron system is obtained from a fictitious non-interacting one, the timedependent Kohn-Sham system, under the action of an effective time-dependent potential. This potential is a functional of the electron density $n(\mathbf{r}, t)$ and, accounts for the non-local spatial and temporal response of the correlated many-body system. ${ }^{39,40}$ As long as collective excitations dominate the optical spectra, simple approximations like adiabatic generalized- 
gradient approximations (AGGA) suffice. ${ }^{35}$ However, the AGGA exhibits problems in the descriptions of the d states, leading, in particular, to d-band energies too close to the Fermi energy, in part due to the incomplete cancellation of the electron self-interaction. The precise description of the delicate interplay between $d$-electron and collective excitations in coinage-metals requires more sophisticated functionals like the long-range-corrected hybrid functional LC-M06L. ${ }^{41,42}$ This problem has likewise been treated successfully using DFT $+\mathrm{U}$ calculations of the generation of hot carriers from surface plasmon decay. ${ }^{43}$

Application of TDDFT is limited to rather small systems having a number of electrons below $\sim 5000$. Therefore, above this size limit we need a more tractable yet robust theoretical framework. Since LSPRs appear naturally as self-sustained solutions of Maxwell's equations, classical local optics provides the required accuracy in many cases. Within this framework, the existence of abrupt metal-dielectric boundaries is assumed, and the electromagnetic response of metallic regions is described in terms of their macroscopic dielectric function, $\epsilon_{\text {bulk }}(\omega)$. In the limit of small sizes and/or sub-nanometric radii of curvature, the non-local nature of the electromagnetic response becomes crucial. ${ }^{13,44}$ As a consequence, the use of spatially dispersive dielectric functions is required for a practical description of the smearing of induced charges across metal-dielectric boundaries. ${ }^{45}$ This can be done through simplified, yet numerically efficient implementations ${ }^{46,47}$ of the hydrodynamical model for electron motion, ${ }^{34,48}$ or by defining a suitable space-dependent dielectric function in the metal-dielectric interface. ${ }^{49}$ These nonlocal corrections lead to blue-shifts of LSPR frequencies with respect to local optics predictions, ${ }^{50,51}$ a trend that can be traced back to the fact that nonlocal corrections spread the induced density inward from the abrupt bulk surface. ${ }^{52}$

While providing relevant corrections to local predictions, nonlocal optics still lacks important ingredients in the elucidation of LSPR behavior. First, the spill-out of the electron density at metal-dielectric interfaces, which in general leads to LSPR frequency red-shifts ${ }^{53,54}$ is missing. Second, quantum finite size effects that are manifest in the discretization of electron energy levels ${ }^{28}$ are absent. Finally, surface corrugation at the sub-nanometric scale 
is essentially missing, although it can be partially accounted for through phenomenological modelling of metal/vacuum interfaces in an effective manner. ${ }^{24,26}$ By contrast the quantum mechanical TDDFT calculations are exact in principle, and would provide all the quantum many-body corrections if it were not for the fact that we need to rely on approximate functionals/kernels for practical calculations. Beyond these approximations, TDDFT can provide all the necessary corrections to local optics results, not only those due to bulk spatial nonlocality in the metal permittivity.

Systems and Methods. Following previous studies on the absorption spectra of noblemetal nanorods, ${ }^{21,22,55}$ we consider model pentagonal rods of $\mathrm{Au}$ and $\mathrm{Ag}$ derived from the 13-atom decahedral cluster by stacking rings of 5 atoms each plus a central atom along the rotational axis (see Figure 1). The longest rod (with highest AR) is made up of $N=145$ atoms while the shortest one (with lowest $\mathrm{AR}$ ) has $N=19$. The structures in our study are the same for both $\mathrm{Au}$ and $\mathrm{Ag}$ and, to allow for a proper discussion of the dependence of the optical absorption on AR, they are not relaxed. The atom-atom distance along the rod axis is set to $d_{0}=2.88 \AA$. The other class of systems here considered are single atom-chains of $\mathrm{Au}$ and $\mathrm{Ag}$ having a number of atoms between 6 and 24, with the same inter-atomic distance $d_{0}$ as in the rods. We focus our investigation on excitations by electric fields polarized along the longitudinal axis ( $x$-direction), and the corresponding absorption spectra are calculated using the time-propagation formalism ${ }^{56}$ of TDDFT. The rods are excited by a delta-impulse perturbing electric field $\delta \mathbf{E}(\mathbf{r}, t)=E_{0} \tau_{0} \delta(t) \mathbf{e}_{x}$, see Figure 1 , and the frequency-dependent absorption cross section is obtained from the Fourier transform of the induced time-dependent dipole moment

$$
\sigma_{\mathrm{abs}}(\omega)=-\frac{4 \pi \omega}{c E_{0} \tau_{0}} \Im \int_{0}^{\infty} d t e^{i \omega t} \int d \mathbf{r} x \delta n(\mathbf{r}, t)
$$

Here, $c$ is the speed of light and $\delta n(\mathbf{r}, t)$ is the time-dependent induced density after the "kick". This induced density is computed through the solution of the time-dependent 
Kohn-Sham equations as implemented in the real-space code octopus. ${ }^{57}$ Valence $s$ - and $d$ electrons are treated explicitly, whereas core electrons are described through norm-conserving Troullier-Martins pseudopotentials. ${ }^{58}$ The Perdew-Burke-Ernzerhof ${ }^{59}$ (PBE) AGGA exchangecorrelation functional is used. However, as anticipated, the performance of this particular functional has to be carefully assessed. Figure S2 in the Supporting Information (SI) shows a comparison of PBE results for selected rods with those obtained using the more sophisticated LC-M06L hybrid functional, obtained by applying a long-range correction ${ }^{60}$ to the meta-GGA functional of Ref. 41. This functional has demonstrated high accuracy for small silver clusters ${ }^{42}$ and is therefore considered as a benchmark here. Our comparison indicates that the PBE spectra are valid as long as the dominant LSPR is well decoupled from interband transitions, which is the case for the high-AR rods treated here. By contrast, their accuracy degrades strongly when LSPRs couple with d-electron excitations, as in the case of $\mathrm{Ag}_{19}$, the shortest silver rod considered here. Thus, special care needs to be taken when comparing TDDFT and electromagnetics predictions for such cases.

A consistent comparison of LSPRs from ab initio and electromagnetics calculations requires a proper correspondence between atomistic structures and geometric regions having well defined boundaries. In order to mimic the soft single-atom termination of our atomistic rods, we choose 'cigar'-shaped geometries for our classical optics metal regions. These sub-nanometric cigars are defined as cylinders of length $a$ and radius $r_{0}$ decorated by two hemispherical ends of the same radius, see Fig. 1. The electron density in the cigars is the same as the average s-electron density in the bulk, given by $n_{\text {avg }}=3 /\left(4 \pi r_{s}^{3}\right)$, where, $r_{s}=3.02 \mathrm{a}_{\mathrm{B}}($ Bohr radius) is the Wigner radius for Au and Ag. As mentioned above, from the electromagnetics perspective, the optical response of noble-metal nanostructures is described through their dispersive bulk permittivity, $\epsilon_{\text {bulk }}(\omega)$. For coinage metals, this consists of a Drude term accounting for the contributions of loosely bound (nearly free) conduction band electrons (s-electrons) and a series of Lorentzian terms describing the effect of strongly bound core electrons. In our calculations, we use experimental fittings of this form and tested 


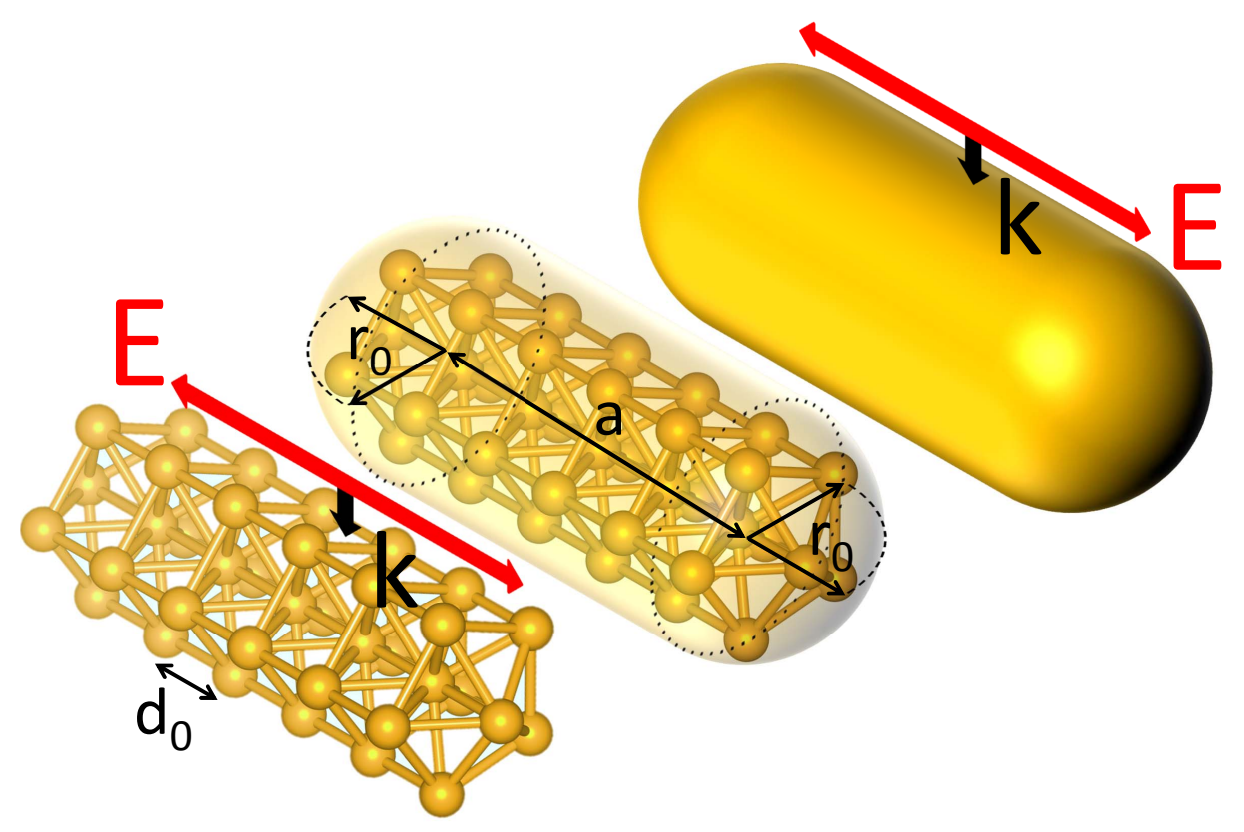

Figure 1: Sketch of the modelling of Ag and Au rods: ionic lattice (left) for TDDFT calculations, and cigar-shaped volume geometry (right) for classical optics. In between, the definition of the boundaries of the rod geometry establishing the mapping between both levels of description is illustrated. The geometrical parameters are indicated: atom-atom distance, $d_{0}$, and the cigar-geometry curvature radius and bulk length, $r_{0}$ and $a$, respectively.

at visible frequencies (up to $5 \mathrm{eV}$ ). ${ }^{61}$ The proper modelling of the atomistic quantum-sized systems is achieved by defining the electromagnetics geometries through two parameters. First, the $\mathrm{AR}=\left(a+2 r_{0}\right) / 2 r_{0}$ for the classical optics cigar is chosen to be the same as for the atomistic structures. Second, their volume, $V$, is set so that the number, N, of Drude electrons (expressed in terms of the fitted parameter for the plasma frequency) is equal to the number of s electrons in the TDDFT calculations. More details on the cigar geometry definition and optimization can be found in the SI.

Both local and non-local optics calculations for the rods' absorption spectra were performed using COMSOL Multiphysics (TM), a commercial solver of Maxwell's Equations which implements the finite element method. The nonlocal spatial dispersion in the metal permittivity is modelled through the so-called hydrodynamic model, with a $\beta$ parameter 
(Fermi velocity) equal to $10^{6} \mathrm{~m} / \mathrm{s} .{ }^{44}$ Within the hydrodynamic model (see Ref. 51 for details of the method and its implementation), bulk longitudinal plasmon modes can be optically excited inside the metal. This leads to an induced surface charge thickening which cannot be described within the realm of local optics. The implementation of the hydrodynamic model is simplified here by using the so-called local analogous model, ${ }^{49}$ which accounts for the smearing of metal boundaries through a fictive dielectric layer of vanishing thickness, $d$, covering the metal surface. The dielectric function within this layer is $\epsilon_{\text {layer }}(\omega)=\frac{\epsilon_{\text {bulk }}(\omega)}{\epsilon_{\text {bulk }}(\omega)-1} q_{\mathrm{L}} d$, where

$$
q_{\mathrm{L}}=\sqrt{-\epsilon_{\mathrm{bulk}}(\omega)} \frac{\omega}{\beta}
$$

is the longitudinal plasmon evanescent wave-vector expressed in terms of the bulk permittivity. Equation (2) gives the dispersion relation of the longitudinal plasmon modes within the bulk metal. In all our nonlocal hydrodynamic calculations, the convergence of results in the limit $d \rightarrow 0$ was checked.

Absorption Spectra. Figure 2 shows a direct comparison between ab initio and local and nonlocal optics absorption spectra for $\mathrm{Ag}$ and $\mathrm{Au}$ sub-nanometric rods. There is an excellent agreement between TDDFT (reported already in Ref. 22 and in general agreement with Ref. 21 \& 55) and local optics calculations for structures with AR > 5 (whose response is dominated by the LSPR). Let us remark that there is no fitting between $a b$ initio and electromagnetics models; only the a priori definition of classical geometries introduced above was followed. The comparison against ab initio predictions improves even further when the absorption cross-section is computed using a nonlocal hydrodynamic metal permittivity. This gives rise to the aforementioned nonlocal resonance blue-shift, a signature of the different LSPR energy dependence on absolute size in local and nonlocal optics. See Figure S3 of the SI for a comprehensive analysis of the dependence of this nonlocal blue-shift on absolute size and AR.

Classical optics fails to accurately describe the ab initio spectra for compact low-AR structures in Figure $2(N \leq 37$ atoms, $\mathrm{AR}<2.9)$. This is expected, since genuine quantum 

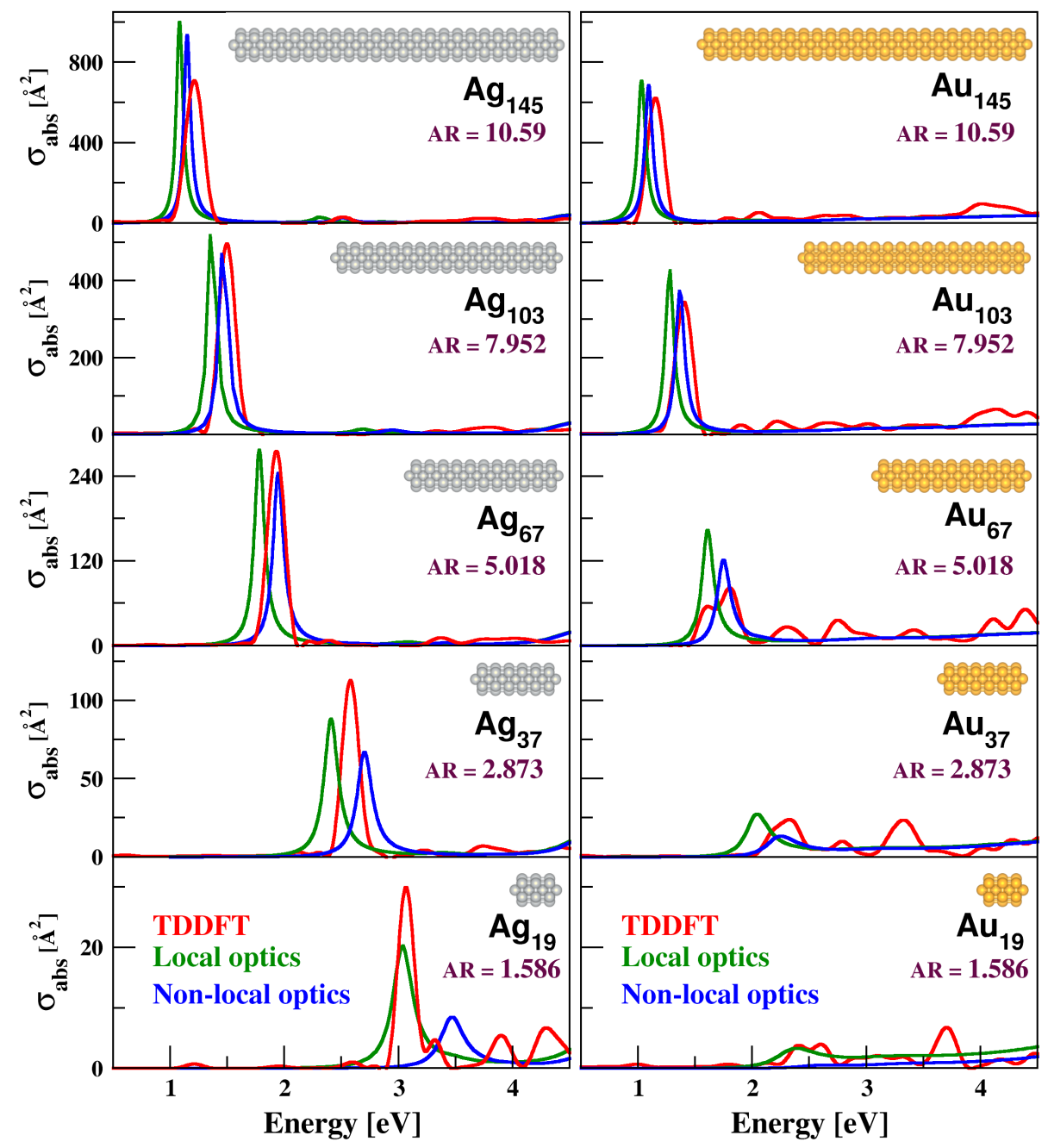

Figure 2: Optical absorption cross section, for silver (left column) and gold (right column) three-dimensional rods of different aspect ratio (AR) and number of atoms.

effects, such as electron-density spill-out and energy level discretization due to size confinement, become more significant as the rod's length (and thus AR) decreases. However, in the case of $\mathrm{Ag}$, despite relatively small spectral deviations, TDDFT and classical calculations are still in qualitative agreement even for low-AR. By contrast, for Au rods having AR $\leq 5.018$ (i.e., $N \leq 67$ atoms), classical predictions are already qualitatively different from the TDDFT spectra. In these Au systems, the classical LSPR frequencies lie at or above the onset of interband transitions $(\sim 2 \mathrm{eV})$ due to d-electrons. This fact prevents the formation of purely collective surface plasmon resonances in the TDDFT spectrum, which in turn is 


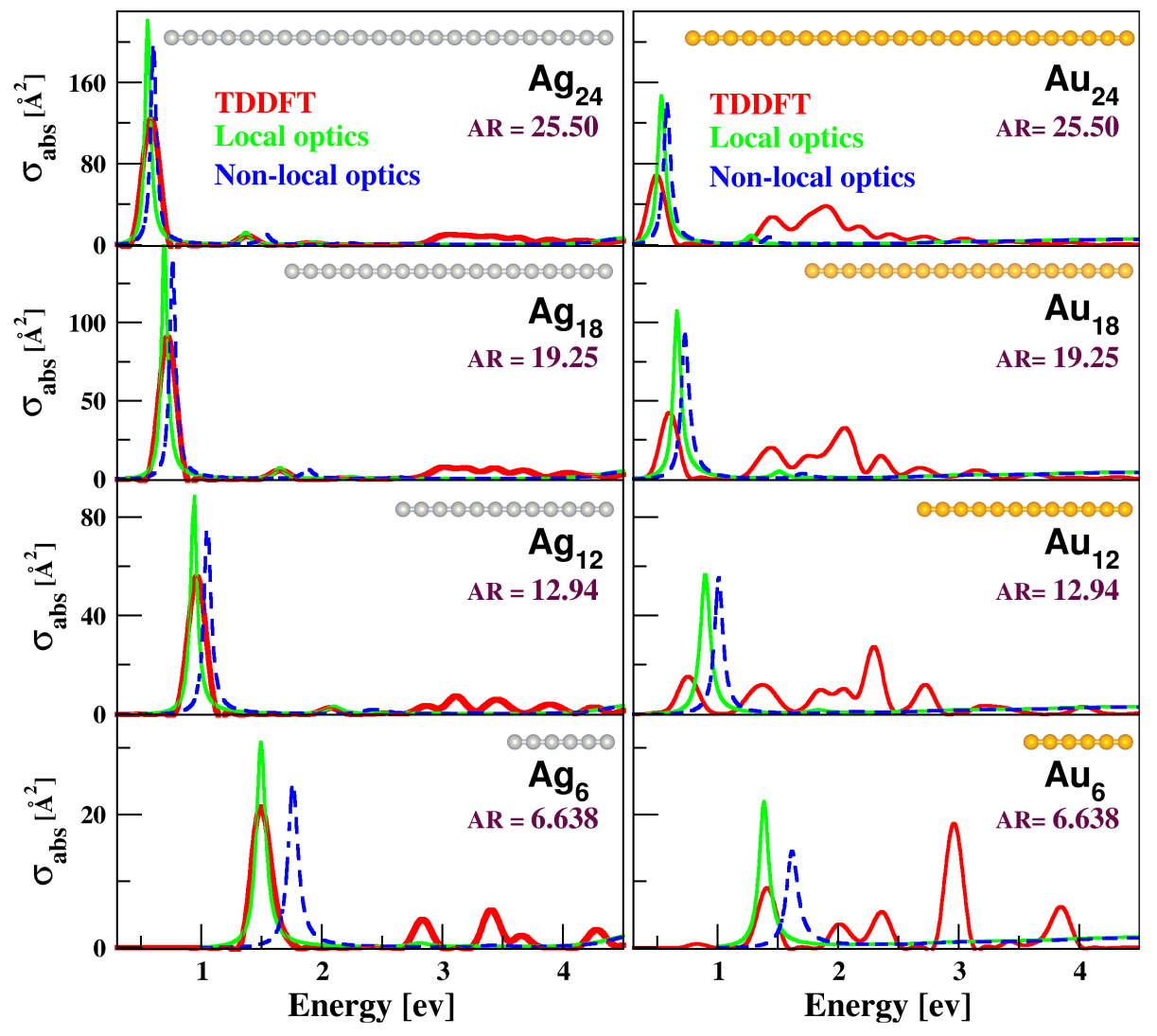

Figure 3: Optical absorption cross section, for quasi-one-dimensional silver (left column) and gold (right column) atomic chains of different aspect ratio (AR) and number of atoms.

dominated by a set of multiple maxima (of similar height) originated by d-electron excitations. Identifying the lowest frequency absorption features in the TDDFT spectra for short $\mathrm{Au}$ rods as LSPRs (having noticed that these are located at roughly the same position as the classical optics peaks), we can conclude that the spectral overlap with the d-excitations gives rise to the partial damping, splitting and fragmentation of LSPRs. It is worth mentioning here that the inclusion of nonlocality in the electromagnetics calculations does not reproduce any of these purely quantum physical features. It only improves the comparison against $a b$ initio results at higher AR by correcting local classical optics LSPR frequencies, which are otherwise too red-shifted due to the abrupt character of induced local charges.

Figure 3 plots the absorption spectra for $\mathrm{Ag}$ and $\mathrm{Au}$ atomic chains of different AR. We find a strikingly good agreement between TDDFT and local optics results for Ag chains, an 
observation similar to previous studies on linear hydrogen atomic chains. ${ }^{62,63}$ As in the case of the rods in Figure 2, for Ag atomic chains the weak spectral deviations above $\sim 3 \mathrm{eV}$ can be attributed to single electron-hole excitations owing to interband transitions. These spectral features are more apparent in Au chains, where the spectral weight of transitions involving d-electrons is comparable to those attributed to LSPRs. As discussed below, the predictive value of local electromagnetics calculations for high AR chains (for both Ag and $\mathrm{Au}$ ) originates from their quasi-one-dimensional nature: electron motion in these systems is confined along the longitudinal axis. Therefore, the collective LSPR that dominates their absorption spectra cannot be regarded as a surface mode. ${ }^{64-67}$ We note that our hydrodynamic model was constructed to account for the impact of spatial nonlocality due to the optical excitation of three-dimensional (bulk) longitudinal plasmons, described by Equation (2), instead of one-dimensional ones. This explains the offset of nonlocal predictions in Figure 3, significantly blue-shifted with respect to local optics and ab initio spectra.

The striking agreement between the absorption spectra for quasi-one-dimensional quantum systems and their three-dimensional local optics counterparts can be easily understood in terms of simple geometrical considerations. To a first approximation, we can take the analytical expression ${ }^{68}$ for the lowest LSPR in ellipsoidal nanoparticles for our cigar geometries. In the limit of large AR, we obtain

$$
\omega_{\mathrm{LSPR}}^{2}=\frac{4}{\pi} \frac{\rho_{\mathrm{avg}} e^{2}}{m_{e} \epsilon_{0}\left(a+2 r_{0}\right)^{2}} \ln (2 \mathrm{AR}),
$$

where $\rho_{\text {avg }}=\pi r_{0}^{2} n_{\text {avg }}$ is the number of Drude electrons per unit length, $\epsilon_{0}$ is the vacuum permittivity, and $m_{e}$ and $e$ the electron mass and charge, respectively. It is worth noticing that the absence of the Lorentzian component of $\epsilon_{\text {bulk }}(\omega)$ in Equation (3) indicates that the dynamical screening by d-electrons is ineffective for rods with large AR. On the other hand, the plasmon dispersion relation in a homogeneous one-dimensional electron system in the long wavelength limit reads ${ }^{23} \omega_{1 \mathrm{D}}^{2}(q)=\frac{\rho_{\text {avg }} e^{2}}{2 \pi \epsilon_{0} m_{e}} q^{2} \ln \left(\xi_{0} q / \pi\right)$, where $\xi_{0}$ is the length parameter 
measuring the electron confinement in the transverse direction. Evaluating the expression above at $q=\pi /\left(a+2 r_{0}\right)$ and making $\xi_{0}=r_{0}$, we obtain the resonant frequency for the dipole collective mode sustained by the system, the ab initio equivalent for the classical LSPR,

$$
\omega_{1 \mathrm{D}-\text { dipole }}^{2}=\frac{\pi}{2} \frac{\rho_{\mathrm{avg}} e^{2}}{m_{e} \epsilon_{0}\left(a+2 r_{0}\right)^{2}} \ln (2 \mathrm{AR})
$$

Equations (3) and (4) describe collective excitations of different nature, but their remarkable similarity allows us to identify both of them. This also clarifies the unexpected agreement between TDDFT and local optics spectra in Figure 3. Note that the equations above yield $\omega_{\text {LSPR }}<\omega_{1 \mathrm{D}-\text { dipole }}$, which is also in agreement with the numerical spectra. Classical optics slightly underestimates the LSPR frequencies in the limit of very long chains (where the analytical expressions are valid), a deviation which, in principle, could be corrected through the appropriate one-dimensional nonlocal corrections.

As mentioned above, the TDDFT spectra for Au chains in Figure 3 indicate that quasione-dimensional collective excitations are not well resolved for $N \leq 12$. In fair correspondence with the three-dimensional case, this effect can be directly attributed to the coupling of the collective mode with single electron-hole excitations. This coupling can be observed in detail in Figure 4, where we show the variation of the absorption cross section normalized to $N$ for different Au chains. For $N=18$, the quasi-one-dimensional LSPR is well separated from the spectral region where single-electron transitions occur. As a consequence, it appears as a well defined absorption peak whose position is in good agreement with the one predicted classically. When decreasing the number of atoms, the lowest frequency maxima lose spectral weight. For $N=10$, this resonance is fragmented and none of the local maxima can be interpreted as the collective LSPR excitation. However, Figure 4 demonstrates that classical optics describes a rough spectral average of the fragmented peaks. Finally, for the 6-atom chain, the lowest-frequency ab initio maximum is very weak, and the transfer of spectral weight to the region of electron-hole excitations is almost complete. Interestingly, a new 


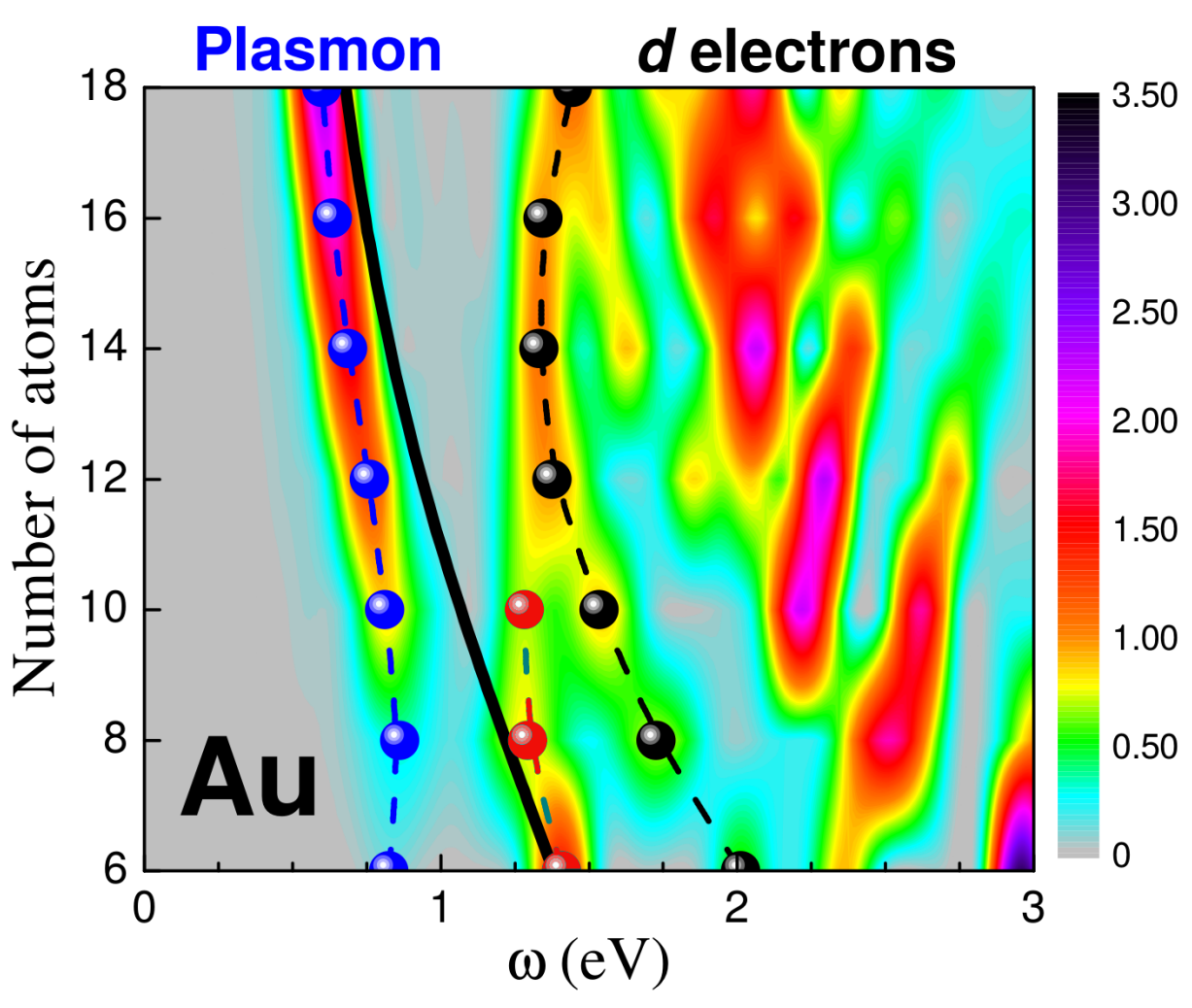

Figure 4: TDDFT absorption cross section per atom (in $\AA^{2}$ ) versus frequency and the number of atoms in Au chains. The solid line plots the LSPR frequency under local optics prescription. The various ab initio spectral maxima are rendered by solid circles (connected as a guide to the eye): collective plasmon modes in blue and single d-electron excitations in black and red.

spectral feature emerges very close to the frequency corresponding to the classical plasmon by taking spectral weight from the original one-dimensional LSPR and the lowest-energy electron-hole excitation. Although plasmon formation in gold chains is clearly beyond the scope of any classical optics treatment, it is worth emphasizing that it seems to account rather accurately for the spectral average of the split quantum excitations in the ab initio calculations.

LSPR frequency versus aspect ratio. As shown above, our combined ab initio and electromagnetics modelling of $\mathrm{Ag}$ and $\mathrm{Au}$ rods indicates that, through a careful mapping between atomistic structures and classical geometries containing a bulk permittivity, LSPRs can be quantitatively described by classical optics methods as long as they are free of inter- 


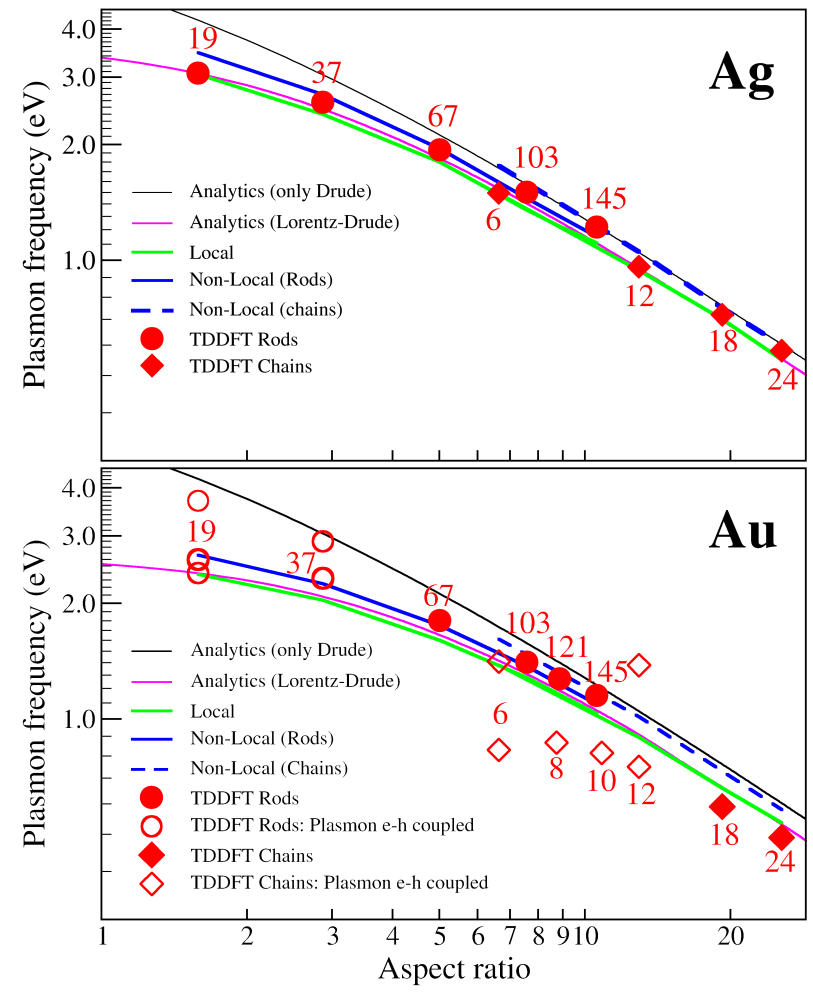

Figure 5: LSPR frequencies in Ag (upper panel) and Au (lower panel) sub-nanometric rods and atomic chains versus aspect ratio. Black (magenta) line renders analytical local optics calculations in ellipsoidal geometries with fitted Drude (Drude-Lorentz) permittivity, where analytics refers to equations $5.13 \mathrm{~b}, 5.15$ of Ref. 6 . Green and blue (solid and dashed) lines plot local and nonlocal classical simulations for Drude-Lorentz cigars, respectively. TDDFT LSPR frequencies for $N$-atom systems are shown in solid red circles (rods) and rhombuses (chains). Empty circles (rods) and rhombuses (chains) correspond to single electron-hole excitations apparent in the absorption spectra.

band contamination. In order to further check this conclusion and provide a more general perspective, we plot in Figure 5 the spectral position of all the absorption maxima in Figures 2, 3 and 4 versus the AR of the various $\mathrm{Ag}$ (top) and Au (bottom) systems (note the log-log scale). TDDFT results for collective LSPR-like modes are shown in solid red circles (rods) and rhombuses (chains). In all cases, the number of atoms is indicated. Significant absorption features in $\mathrm{Au}$ structures originating from single electron-hole excitations are represented by empty circles (rods) and rhombuses (chains). Local (nonlocal) optics predictions for the corresponding cigar geometries are rendered in green (blue) lines. We observe that while the LSPR frequencies within the local approximation depend only on AR, they depend 
on absolute size once nonlocal corrections are included. Thus, solid and dashed blue lines correspond to nonlocal resonances for rods and chains, respectively.

Figure 5 shows that while the AR-dependence of LSPR frequencies obtained from local optics calculations is already in good agreement with TDDFT results, the non-local blue-shift slightly improves it for long Au and Ag rods. This suggests that these systems, despite having lateral sub-nanometric dimensions, preserve a quasi-bulk optical response. By contrast, in the Ag chains, the TDDFT resonant frequencies are better described within the local optics frame. In the top panel of Figure 5, circles lie above rhombuses, which indicates that the rod LSPRs are blue-shifted with respect to the atomic chain ones. This trend is exactly the opposite to that predicted by nonlocal optics (see solid and dashed blue lines). As discussed above, not only this failure of the nonlocal hydrodynamic model, but also the good agreement between local optics and ab initio predictions in Ag chains can be linked to the inherent onedimensional nature of these systems. In the case of short $\mathrm{Au}$ rods and chains, the occurrence of d-electron transitions above $\sim 2 \mathrm{eV}$ causes qualitative differences in the spectral behavior in TDDFT and in electromagnetics approaches. The discrepancy in the AR dependence between both methods is most apparent in chains with $N=6-12$, where multiple ab initio single-electron peaks occur far apart from the LSPR band. In order to verify the validity of local optics descriptions, we evaluate the ellipsoidal LSPR dispersion in Ref. 68 for the full Drude-Lorentz permittivity as well as for a simplified Drude-only fitting. The results, plotted in magenta (full $\epsilon_{\text {bulk }}(\omega)$, i.e. Drude and Lorentz parts) and black (simplified $\epsilon_{\text {bulk }}(\omega)$, i.e. Drude part only), in Figure 5 lower panel reveal that agreement with TDDFT predictions occurs if the dielectric function is mainly governed by the Drude contribution, as expected.

Induced density. To gain further insight into our results, we compare now the near-field characteristics of LSPRs, interpreted as collective dipolar surface modes, within the ab initio and classical optics frameworks. Specifically, we consider the cross-sectional integration of the resonant induced charge densities along the rod length (for 3D visualization cf., Ref. 69). This magnitude can be evaluated using TDDFT by exciting the atomistic systems 

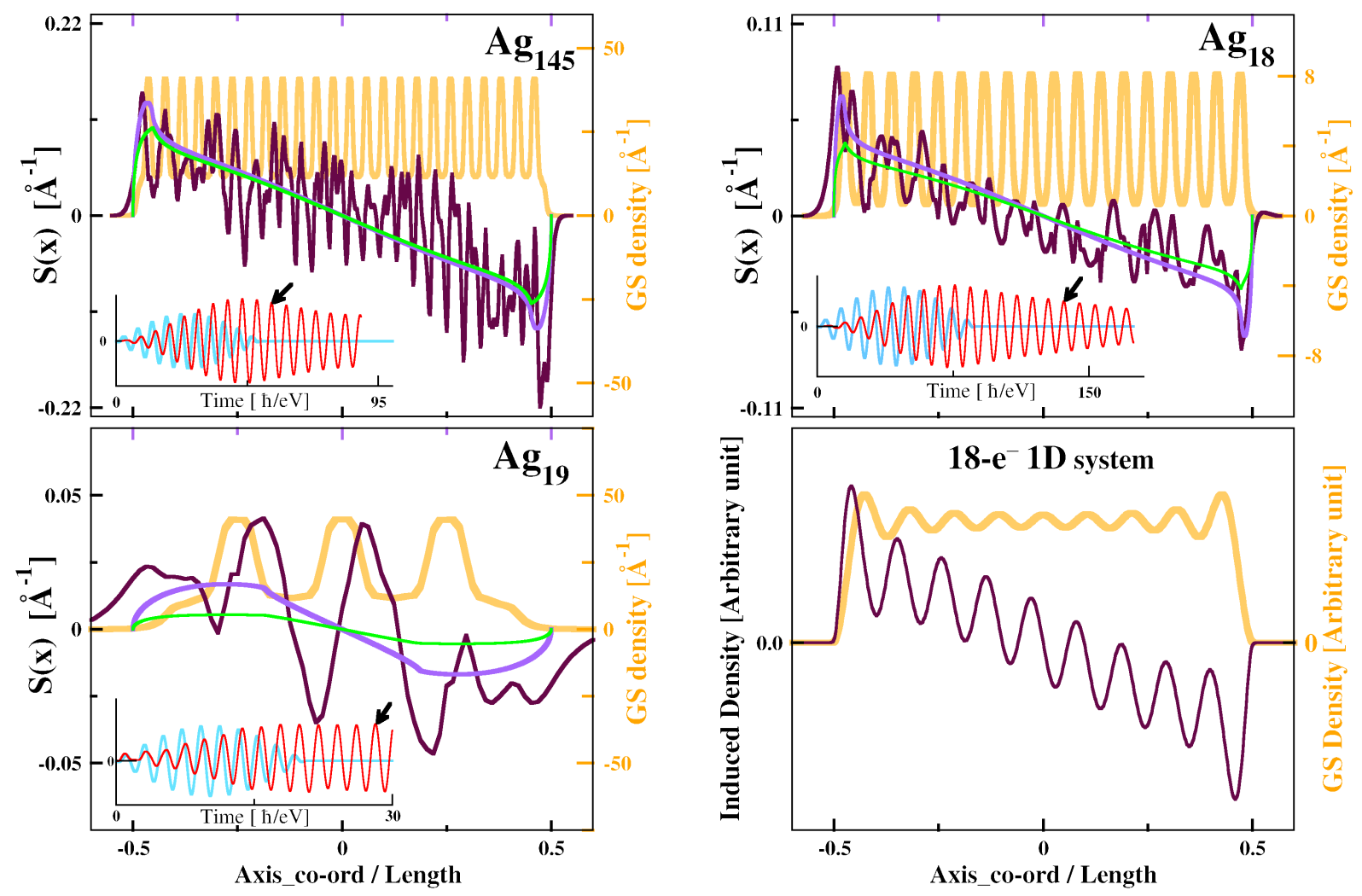

Figure 6: Induced charge density for $\mathrm{Ag}_{145}$ (top left), $\mathrm{Ag}_{19}$ (bottom left), $\mathrm{Ag}_{18}$ (top right), and a $N=18$ purely one-dimensional system (bottom right). In all panels, the brown curve plots a snapshot of the TDDFT induced charge density along the normalized rod/chain length in units of electrons/A. The orange curve renders the ground-state density profile, shown for reference. The violet and green curves in the first three panels show the induced surface charge distribution (in electrons $/ \AA$ ) obtained from local and non-local optics calculations, respectively. The insets show the time dependence of the driving laser (blue) and of the TDDFT induced dipole moment (red).

with a weak quasi-monochromatic laser field polarized along the rod axis ( $x$-direction). The laser pulse has a sinusoidal envelope of finite duration, $T$, and its bandwidth is centered at resonance with the LSPR frequency: $\mathbf{E}(t)=E_{0} e^{i \omega_{\mathrm{LSPR}}{ }^{t}} \sin \left(\omega_{\mathrm{LSPR}} t / T\right) \mathbf{e}_{x}$. In our calculations the envelope duration was set to 10 plasmon oscillations $\left(T=20 \pi / \omega_{\mathrm{LSPR}}\right)$. The system is left evolving freely for a sufficient amount of time after the external driving laser is switched off. The cross-sectional-integrated charge density can be then evaluated from

$$
S(x)=\iint\left[\rho(x, y, z ; \tau)-\rho_{G S}(x, y, z)\right] d y d z
$$


where, $\rho_{G S}(x, y, z)$ is the ground-state density and $\rho(x, y, z ; \tau)$ is the time-dependent density at a time $\tau$, longer than $T$, when the oscillation of the electron density is self-sustained. Within the ab initio description, the induced charges spread across the rod's volume. By contrast, in the electromagnetics picture, they are confined at the metal surface: surface charges are located exactly at the geometric boundaries in the local optics treatment, whereas nonlocal corrections provide the charge distribution with a non-vanishing thickness. Taking this into account, we can estimate the induced charges using classical electromagnetics through the discontinuity in the normal component of the electric field. Explicitly, we can write

$$
S(x)=2 \pi \epsilon_{0} r(x)\left[\mathbf{E}_{\text {out }}(x)-\mathbf{E}_{\text {in }}(x)\right] \cdot \mathbf{e}(x)
$$

where, $r(x)=\sqrt{y(x)^{2}+z(x)^{2}}$ is the radial coordinate along the metal boundary, $\mathbf{e}(x)$ is the unit vector along the surface normal, and $\mathbf{E}_{\text {out }}(x)$ and $\mathbf{E}_{\text {in }}(x)$ are the electric fields outside and inside the cigar boundaries. Note that, taking into account the confined (extended) character of induced surface charges, $\mathbf{E}_{\text {in }}(x)$ is evaluated exactly at (at a distance $1 / q_{\mathrm{L}}$ from) the metal-dielectric interface in the local (nonlocal) calculations.

Figure 6 plots $S(x)$ against the length-normalized axis co-ordinate for three distinct Ag systems whose spectra (both in TDDFT-PBE and in classical optics) present a well defined LSPR: 145-atom rod (top left panel), 19-atom rod (bottom left panel) and $N=18$ atomic chain (top right panel). In all cases, the induced density profiles obtained from TDDFT (brown), and local (violet) and non-local (green) classical optics are shown. The TDDFT ground-state density is shown as orange line (note the different scales of the left and right axes in all panels). To allow for a quantitative comparison between ab initio and electromagnetics treatments of $S(x)$, the amplitude of the driving laser in all simulations was set to $10^{8} \mathrm{~V} / \mathrm{m}$ (within the linear response). The insets show the laser field (blue) and the TDDFT time evolution of the induced dipole moment (red). The black arrows indicate the time $\tau$ at which the induced charges were computed. The bottom right panel renders the ab initio induced 
(brown) and ground state (orange) density profiles for a 18 electron purely one-dimensional jellium system modelling $\mathrm{Ag}_{18}$.

Figure 6 shows that the excellent quantitative agreement between $a b$ initio and classical predictions for the absorption spectra of $\mathrm{Ag}_{145}$ (see Figure 2) and $\mathrm{Ag}_{18}$ (Figure 3) also holds in the near-field regime. Remarkably, TDDFT and electromagnetics induced charge peaks are not only located at the same position but present very similar heights. This can be interpreted as a consequence of the high plasmonic character of both structures, which support well-defined collective LSPRs free of the contamination from single electron-hole excitations. There are two relevant aspects in the $a b$ initio density profiles which deserve attention. First, there is the inhomogeneity of $S(x)$ across the length of the structure. These regular modulations of the density are caused by the highly localized nature of the d-electrons and their contribution to the overall response of the system. Second, there are weak, somewhat irregular fluctuations overlaid onto those modulations. These arise from the fact that the quasi-monochromatic laser excitation has a finite energetic width and hence can excite several energetically close modes. The superposition of the different modes leads to the irregular fluctuations which modify the overall behavior of the induced density and lead to a certain asymmetry in the density profile. By comparison, a static external field would lead to a profile reflecting the perfectly symmetric geometry along the rod axis. Both, the modulations due to the atomistic density inhomogeneity and the fluctuations due to the superposition of different modes are naturally absent in the classical induced-density profiles. The right bottom panel of Figure 6 confirms our interpretation of these TDDFT induced density features missing in the classical optics results. It renders a perfectly symmetric, and smoothly oscillating $S(x)$ (free from sharp fluctuations) for the purely one-dimensional jellium system.

Despite the correspondence in the LSPR spectral position (see lowest left panel in Figure 2), the comparison between classical and ab initio induced densities for $\mathrm{Ag}_{19}$ (shown in the left bottom panel of Figure 6) is rather poor. Unlike $\mathrm{Ag}_{145}$ and $\mathrm{Ag}_{18}$, electromagnetic cal- 
culations for $\mathrm{Ag}_{19}$ yield a $S(x)$ profile which is not peaked at the rod ends. On the contrary, induced charges spread significantly along the nanorod length towards its middle plane. The TDDFT distribution develops several maxima along the length of the system. These are reminiscent of the sharp fluctuations in the ab initio $S(x)$ for $\mathrm{Ag}_{145}$ and $\mathrm{Ag}_{18}$. This indicates that, as expected from the high LSPR frequency apparent in its absorption spectrum $(\sim 3$ $\mathrm{eV}$, d-electron transitions play a key role in the optical response of $\mathrm{Ag}_{19}$. Thus, we can conclude that this rod configuration presents a rather low plasmonic character, despite the fact that its TDDFT-PBE absorption spectrum is dominated by a single peak reproduced by classical optics. In fact, as mentioned before, our TDDFT calculations use the PBE AGGA functional, whose validity for the description of $\mathrm{Ag}_{19}$ is questionable. This appears in a refined ab initio study on $\mathrm{Ag}_{19}$, comparing PBE with the range-separated hybrid functional LC-M06L, ${ }^{41,60}$ which we have included in the SI (Figure S2). The LC-M06L functional is more suitable for the treatment of d-electron excitations and yields accurate spectra for small Ag clusters. ${ }^{42}$ From our analysis, we conclude that TDDFT-PBE calculations for $\mathrm{Ag}_{19}$ are not reliable, and hence, the spectral agreement between classical and fully quantum results on $\mathrm{Ag}_{19}$ is partially coincidental. In fact, the comparison of $a b$ initio and classical LSPR induced densities for $\mathrm{Ag}_{19}$ (see Figure 6) establishes the limitations of the electromagnetics predictions. They fail to describe structures in which LSPRs and d-electrons couple strongly. Even in the TDDFT treatment of those systems, a description of exchange and correlation better than the AGGA functionals is required. In contrast, if the LSPR is largely free of d-electron excitations (as it is for $\mathrm{Ag}_{145}$ and other long rods and atomic chains), the ab initio spectra obtained using PBE AGGA functionals are accurate (see Figure S2 of SI). In this situation, classical optics predicts remarkably well the TDDFT spectral position, strength and character of the induced LSPR density.

Conclusions. In conclusion, we have presented a comprehensive theoretical study on the plasmonic properties of sub-nanometric elongated coinage-metal systems. Our investigation compares local and nonlocal classical optics and quantum ab initio methods. By 
preserving the aspect ratio and number of Drude (or s) electrons, we have established simple model geometries, which can be treated within the electromagnetics framework, in correspondence with atomistic structures having sub-nanometric dimensions. We find a remarkable agreement between the ab initio and classical descriptions of the absorption spectral characteristics of Ag rods and chains. The agreement for the equivalent Au systems is significantly worse due to the low-energy onset of d-electron excitations. In addition, we have shown that it is the shape, not the overall size, which determines the degree of d-electron contamination suffered by the localized plasmon resonances sustained by these structures. Finally, we have transferred our comparative analysis from the far-field to the near-field regime. We have demonstrated that, despite its higher sensitivity to atomistic features, the classical and the fully quantum mechanical descriptions of localized plasmon-induced charges are in remarkable agreement for elongated Ag systems.

Supporting Information. A detailed description of the mapping between atomistic structures and classical electromagnetics geometries is presented in Section I. Section II shows the comparison between TDDFT absorption spectra obtained using PBE GGA and LC-M06L functionals for three different Ag rods. Finally, an study of the dependence of the nonlocal optics blue-shift on aspect ratio and absolute size of Ag rods is provided in Section III. This material is available online free of charge via http://pubs.acs.org.

\section{Acknowledgement}

This work has been carried out thanks to the support of the A*MIDEX grant ( ${ }^{\circ}$ ANR11-IDEX-0001-02) funded by the French Government "Investissements d'Avenir" program. We acknowledge support from the French National Research Agency (Agence Nationale de Recherche, ANR) in the frame of the project "FIT SPRINGS", ANR-14-CE08-0009. This work has used HPC resources from GENCI-IDRIS (Grant 2016-096829). PGG and AIFD acknowledge funding from the Spanish MINECO through the "María de Maeztu" programme 
for Units of Excellence in R\&D (MDM-2014-0377) and through research grants MAT201453432-C5-5-R (PGG) and FIS2015-64951-R (AIFD). AIFD also acknowledges funding from the EU Seventh Framework Programme under contract FP7-PEOPLE-2013-CIG-630996.

\section{References}

(1) Anker, J. N.; Paige-Hall, W.; Lyandres, O.; Shah, N. C.; Zhao, J.; Van-Duyne, R. P. Biosensing with plasmonic nanosensors. Nature Materials 2008, 7, 442-453.

(2) Seney, C. S.; Gutzman, B. M.; Goddard, R. H. Correlation of Size and Surface-Enhanced Raman Scattering Activity of Optical and Spectroscopic Properties for Silver Nanoparticles. J. Phys. Chem. C 2009, 113, 74-80.

(3) Bardhan, R.; Lal, S.; Joshi, A.; Halas, N. J. Theranostic Nanoshells: From Probe Design to Imaging and Treatment of Cancer. Acc. Chem. Res. 2011, 44, 936-946.

(4) Knight, M. W.; Sobhani, H.; Nordlander, P.; Halas, N. J. Photodetection with Active Optical Antennas. Science 2011, 332, 702-704.

(5) Novotny, L.; van Hulst, N. Antennas for light. Nat. Photonics 2011, 5, 83-90.

(6) Maier, S. A. Plasmonics: Fundamentals and Applications; Springer: New York, 2007.

(7) Pelton, M.; Bryant, G. Metal-nanoparticle Plasmonics; Wiley: Hoboken, 2013.

(8) Link, S.; El-Sayed, M. A. Spectral Properties and Relaxation Dynamics of Surface Plasmon Electronic Oscillations in Gold and Silver Nanodots and Nanorods. J. Phys. Chem. B 1999, 103, 8410-8426.

(9) Jakab, A.; Rosman, C.; Khalavka, Y.; Becker, J.; Trügler, A.; Hohenester, U.; Sönnichsen, C. Highly Sensitive Plasmonic Silver Nanorods. ACS Nano 2011, 5, 68806885. 
(10) Fang, Y.; Chang, W.-S.; Willingham, B.; Swanglap, P.; Dominguez-Medina, S.; Link, S. Plasmon Emission Quantum Yield of Single Gold Nanorods as a Function of Aspect Ratio. ACS Nano 2012, 6, 7177-7184.

(11) Lermé, J.; Palpant, B.; B., P.; Pellarin, M.; Treilleux, M.; Vialle, J. L.; Perez, A.; Broyer, M. Quenching of the Size Effects in Free and Matrix-Embedded Silver Clusters. Phys. Rev. Lett. 1998, 80, 5105-8.

(12) Cottancin, E.; Celep, G.; Lermé, J.; Pellarin, M.; Huntzinger, J.; Vialle, J.; Broyer, M. Optical Properties of Noble Metal Clusters as a Function of the Size: Comparison between Experiments and a Semi-Quantal Theory. Theor. Chem. Acc. 2006, 116, 51423.

(13) Scholl, J. A.; Koh, A. L.; Dionne, J. A. Quantum plasmon resonances of individual metallic nanoparticles. Nature 2012, 483, 421-7.

(14) Takahata, R.; Yamazoe, S.; Warakulwit, C.; Limtrakul, J.; Tsukuda, T. Rayleigh Instability and Surfactant-Mediated Stabilization of Ultrathin Gold Nanorods. J. Phys. Chem. C 2016, 120, 17006-17010.

(15) Walter, M.; Moseler, M.; Whetten, R. L.; Häkkinen, H. A 58-Electron SuperatomComplex Model for The Magic Phosphine-Protected Gold Clusters (Schmid-gold, Nanogold of 1.4-nm Dimension. Chem. Sci. 2011, 2, 1583.

(16) Nimmala, P. R.; Yoon, B.; Whetten, R. L.; Landman, U.; Dass, A. Au67(SR)35 Nanomolecules: Characteristic Size-Specific Optical, Electrochemical, Structural Properties and First-Principles Theoretical Analysis. J. Phys. Chem. A 2013, 117, 504.

(17) Negishi, Y.; Nakazaki, T.; Malola, S.; Takano, S.; Niihori, Y.; Kurashige, W.; Yamazoe, S.; Tsukuda, T.; Hkkinen, H. A Critical Size for Emergence of Nonbulk Electronic and Geometric Structures in Dodecanethiolate-Protected Au Clusters. Journal of the American Chemical Society 2015, 13\%, 1206-1212, PMID: 25549276. 
(18) Weissker, H.-C.; Escobar, H. B.; Thanthirige, V. D.; Kwak, K.; Lee, D.; Ramakrishna, G.; Whetten, R.; López-Lozano, X. Information on Quantum States Pervades the Visible Spectrum of the Ubiquitous Au144 Gold Nanocluster. Nat. Commun. 2014, 5,3785 .

(19) Weissker, H.-C.; Lopez-Acevedo, O.; Whetten, R. L.; López-Lozano, X. Optical Spectra of the Special Au144 Gold-Cluster Compounds: Sensitivity to Structure and Symmetry. The Journal of Physical Chemistry C 2015, 119, 11250-11259.

(20) Zuloaga, J.; Prodan, E.; Nordlander, P. Quantum Plasmonics: Optical Properties and Tunability of Metallic Nanorods. ACS Nano 2010, 4, 5269-5276, PMID: 20698558.

(21) Guidez, E. B.; Aikens, C. M. Diameter Dependence of the Excitation Spectra of Silver and Gold Nanorods. J. Chem. Phys. C 2013, 117, 12325-12336.

(22) López Lozano, X.; Barron, H.; Mottet, C.; Weissker, H.-C. Aspect-ratio- and sizedependent emergence of the surface-plasmon resonance in gold nanorods - an ab initio TDDFT study. Phys. Chem. Chem. Phys. 2014, 16, 1820-1823.

(23) Sarma, S. D.; Lai, W. Screening and elementary excitations in narrow-channel semiconductor microstructures. Phys. Rev. B 1985, 32, 1401-1404.

(24) Barbry, M.; Koval, P.; Marchesin, F.; Esteban, R.; Borisov, A. G.; Aizpurua, J.; Sánchez-Portal, D. Atomistic Near-Field Nanoplasmonics: Reaching Atomic-Scale Resolution in Nanooptics. Nano Letters 2015, 15, 3410-3419.

(25) Benz, F.; Schmidt, M. K.; Dreismann, A.; Chikkaraddy, R.; Zhang, Y.; Demetriadou, A.; Carnegie, C.; Ohadi, H.; de Nijs, B.; Esteban, R.; Aizpurua, J.; Baumberg, J. Single-molecule optomechanics in picocavities. Science 2016, 354, 726-729.

(26) Trautmann, S.; Aizpurua, J.; Götz, I.; Undisz, A.; Dellith, J.; Schneidewing, H.; Ret- 
tenmayr, M.; Deckert, V. A classical description of subnanometer resolution by atomic features in metallic structures. Nanoscale 2017, 9, 391-401.

(27) Liebsch, A. Surface-plasmon dispersion and size dependence of Mie resonance: Silver versus simple metals. Phys. Rev. B 1993, 48, 11317-11328.

(28) Monreal, R. C.; Antosiewicz, T. J.; Appell, S. P. Competition between Surface Screening and Size Quantization for Surface Plasmons in Nanoparticles. New Journal of Physics 2013, 15, 083044.

(29) Weissker, H.-C.; Mottet, C. Optical properties of pure and core-shell noble-metal nanoclusters from TDDFT: The influence of the atomic structure. Phys. Rev. B 2011, 84, 165443.

(30) Ravishankar, S.; Narang, P.; Jermyn, A. S.; Goddard III, W. A.; Atwater, H. A. Theoretical predictions for hot-carrier generation from surface plasmon decay. Nature Comm. 2014, 5, 5788 .

(31) Mukherjee, S.; Libisch, F.; Large, N.; Neumann, O.; Brown, L. V.; Cheng, J.; Lassiter, J. B.; Carter, E. A.; Nordlander, P.; Halas, N. J. Hot Electrons Do the Impossible: Plasmon-Induced Dissociation of H2 on Au. Nano Lett. 2013, 13, 240-247.

(32) Baffou, G.; Quidant, R. Thermo-plasmonics: using metallic nanostructures as nanosources of heat. Laser Photon. Rev. 2013, 7, 171-187.

(33) Runge, E.; Gross, E. K. U. Density-Functional Theory for Time-Dependent Systems. Phys. Rev. Lett. 1984, 52, 997.

(34) Pitarke, J. M.; Silkin, V. M.; Chulkov, E. V.; Echenique, P. M. Theory of Surface Plasmons and Surface-Plasmon Polaritons. Rep. Prog. Phys. 2007, 70, 1.

(35) Varas, A.; García-González, P.; Feist, J.; García-Vidal, F. J.; Rubio, A. Quantum plasmonics: from jellium models to ab initio calculations. Nanophotonics 2016, 5, 409. 
(36) Sinha-Roy, R.; López-Lozano, X.; Whetten, R. L.; García-González, P.; Weissker, H.-C. In Search of the Quantum-Electronic Origin of Color Change: Elucidation of the Subtle Effects of Alloying with Copper on $1.8 \mathrm{~nm}$ Gold Nanoclusters. The Journal of Physical Chemistry C 2017, 121, 5753-5760.

(37) Barcaro, G.; Sementa, L.; Fortunelli, A.; Stener, M. Optical properties of nanoalloys. Phys. Chem. Chem. Phys. 2015, 17, 27952-27967.

(38) Rossi, T. P.; Lehtola, S.; Sakko, A.; Puska, M. J.; Nieminen, R. M. Nanoplasmonics simulations at the basis set limit through completeness-optimized, local numerical basis sets. The Journal of Chemical Physics 2015, 142, 094114.

(39) Onida, G.; Reining, L.; Rubio, A. Electronic Excitations: Density-Functional Versus Many-Body Greens-Function Approaches. Rev. Mod. Phys. 2002, 74, 601.

(40) Marques, M. A. L., Maitra, N. T., Nogueira, F. M. S., Gross, E. K. U., Rubio, A., Eds. Fundamentals of Time-Dependent Density Functional Theory; Springer: Berlin, 2012.

(41) Zhao, Y.; Truhlar, D. G. A new local density functional for main-group thermochemistry, transition metal bonding, thermochemical kinetics, and noncovalent interactions. J. Chem. Phys. 2006, 125, 194101.

(42) Rabilloud, F. Description of Plasmon-like band in Silver Clusters: The Importance of the Long-range Hartree-Fock Exchange in Time-Dependent Density-Functional Theory Simulations. J. Chem. Phys. 2014, 141, 144302.

(43) Sundararaman, R.; Narang, P.; Jermyn, A. S.; Goddard III, W. A.; Atwater, H. A. Theoretical predictions for hot-carrier generation from surface plasmon decay. Nature Communications 2014, 5, $5788 \mathrm{EP}-$-, Article.

(44) Ciraci, C.; Hill, R. T.; Mock, J. J.; Urzhumov, Y.; Fernández-Domínguez, A. I.; 
Maier, S. A.; Pendry, J. B.; Chilkoti, A.; Smith, D. R. Probing the Ultimate Limits of Plasmonic Enhancement. Science 2012, 337, 1072-1074.

(45) Boardman, A. Electromagnetic Surface Modes. Hydrodynamic Theory of PlasmonPolaritons on Plane Surfaces; John Wiley and Sons: Chichester, 1982.

(46) García de Abajo, F. J. Nonlocal Effects in the Plasmons of Strongly Interacting Nanoparticles, Dimers, and Waveguides. J. Phys. Chem C 2011, 112, 17983-17987.

(47) Raza, S.; Toscano, G.; Jauho, A.-P.; Wubs, M.; Mortensen, N. A. Unusual Resonances in Nanoplasmonic Structures due to Nonlocal Response. Phys. Rev. B 2011, $84,121412(\mathrm{R})$

(48) Eguiluz, A.; Quinn, J. Hydrodynamic model for surface plasmons in metals and degenerate semiconductors. Phys. Rev. B 1976, 14, 1347.

(49) Luo, Y.; Fernández-Domínguez, A. I.; Wiener, A.; Maier, S. A.; Pendry, J. B. Surface Plasmons and Nonlocality: A Simple Model. Phys. Rev. Lett. 2013, 111, 093901.

(50) Stella, L.; Zhang, P.; García-Vidal, F. J.; Rubio, A.; García-González, P. Performance of Nonlocal Optics When Applied to Plasmonic Nanostructures. J. phys. Chem. C 2013, $117,8941-8949$.

(51) Ciraci, C.; Pendry, J. B.; Smith, D. R. Hydrodynamic Model for Plasmonics: A Macroscopic Approach to a Microscopic Problem. Chem. Phys. Chem. 2013, 14, 1109-1116.

(52) Feibelman, P. J. Surface electromagnetic fields. Prog. Surf. Sci. 1982, 12, 287-407.

(53) Toscano, G.; Straubel, J.; Kwiatkowski, A.; Rockstuhl, C.; Evers, F.; Xu, H.; Mortensen, N. A.; Wubs, M. Resonance shifts and spill-out effects in self-consistent hydrodynamic nanoplasmonics. Nature Comm. 2010, 4, 5269-5276.

(54) Ciraci, C.; Della Sala, F. Quantum hydrodynamic theory for plasmonics: Impact of the electron density tail. Physical Review B 2016, 93, 205405. 
(55) Johnson, H. E.; Aikens, C. M. Electronic Structure and TDDFT Optical Absorption Spectra of Silver Nanorods. J. Phys. Chem. A 2009, 113, 4445-4450.

(56) Yabana, K.; Bertsch, G. F. Time-dependent local-density approximation in real time. Phys. Rev. B 1996, 54, 4484-4487.

(57) Marques, M. A.; Castro, A.; Bertsch, G. F.; Rubio, A. Octopus: a first-principles tool for excited electronion dynamics. Comput. Phys. Commun. 2003, 151, 60-78.

(58) Troullier, N.; Martins, J. L. Efficient pseudopotentials for plane-wave calculations. Phys. Rev. B 1991, 43, 1993-2006.

(59) Perdew, J. P.; Burke, K.; Ernzerhof, M. Generalized Gradient Approximation Made Simple. Phys. Rev. Lett. 1996, 77, 3865-3868.

(60) Ikura, H.; Tsuneda, T.; Yanai, T.; Hirao, K. A long-range correction scheme for generalized-gradient-approximation exchange functionals. J. Chem. Phys. 2001, 115, $3540-3544$.

(61) Rakić, A. D.; Djurišić, A. B.; Elazar, J. M.; Majewski, M. L. Optical properties of metallic films for vertical-cavity optoelectronic devices. Appl. Opt. 1998, 37, 52715283.

(62) DePrince III, A. E.; Pelton, M.; Guest, J. R.; Gray, S. K. Emergence of Excited-State Plasmon Modes in Linear Hydrogen Chains from Time-Dependent Quantum Mechanical Methods. Phys. Rev. Lett. 2011, 107, 196806.

(63) Zhang, R.; Zhang, Y.; Dong, Z. C.; Jiang, S.; Zhang, C.; Chen, L. G.; Zhang, L.; Liao, Y.; Aizpurua, J.; Luo, Y.; Yang, J. L.; Hou, J. G. Chemical mapping of a single molecule by plasmon-enhanced Raman scattering. Nature 2013, 498, 82-86.

(64) Gao, S.; Yuan, Z. Emergence of collective plasmon excitation in a confined onedimensional electron gas. Phys. Rev. B 2005, 72, 121406. 
(65) Yan, J.; Gao, S. Plasmon resonances in linear atomic chains: Free-electron behavior and anisotropic screening of d electrons. Physical Review B 2008, 78, 235413.

(66) Bernadotte, S.; Evers, F.; Jacob, C. R. Plasmons in molecules. J. Phys. Chem. C 2013, $117,18631878$.

(67) Wu, R.; Yu, Y.; Xue, H.; Hu, H.; Liu, Q. Dipole and quadrupole plasmon in confined quasi-one dimensional electron gas systems. Physics Letters A 2014, 378, 2995-3000.

(68) Bohren, C. F.; Huffman, D. R. Absorption and Scattering of Light by Small Particles ; Wiley-VCH: New York, 1998.

(69) Weissker, H.-C.; López-Lozano, X. Surface plasmons in quantum-sized noble-metal clusters: TDDFT quantum calculations and the classical picture of charge oscillations. Phys. Chem. Chem. Phys. 2015, 17, 28379-28386. 


\section{Graphical TOC Entry}

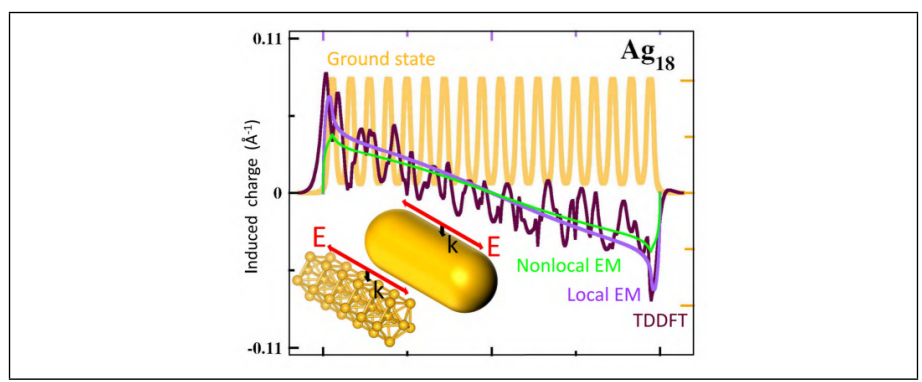

